\title{
RESTRICTION OF THE COTANGENT BUNDLE TO ELLIPTIC CURVES AND HILBERT-KUNZ FUNCTIONS
}

\author{
HOLGER BRENNER AND GEORG HEIN
}

\begin{abstract}
We describe the possible restrictions of the cotangent bundle $\Omega_{\mathbb{P}^{N}}$ to an elliptic curve $C \subset \mathbb{P}^{N}$. We apply this in positive characteristic to the computation of the Hilbert-Kunz function of a homogeneous $R_{+}$primary ideal $I \subset R$ in the graded section ring $R=\bigoplus_{n \in \mathbb{N}} \Gamma(C, \mathcal{O}(n))$.
\end{abstract}

Mathematical Subject Classification (2000): 13A35; 13D02; 13D40; 14H60

\section{INTRODUCTION}

The aim of this paper is to compute the Hilbert-Kunz function of homogeneous primary ideals in the homogeneous coordinate ring or in the graded section ring of an elliptic curve $C \subset \mathbb{P}^{N}$. Recall that for a primary ideal $I$ in a local Noetherian ring $(R, \mathfrak{m})$ or an $\mathbb{N}$-graded algebra $\left(R, R_{+}\right)$of dimension $d$ of positive characteristic $p$ the Hilbert-Kunz function is given by

$$
e \mapsto \lambda\left(R / I^{\left[p^{e}\right]}\right),
$$

where $\lambda$ denotes the length. The Hilbert-Kunz multiplicity of $I$ is defined as the limit

$$
e_{H K}(I)=\lim _{e \rightarrow \infty} \lambda\left(R / I^{\left[p^{e}\right]}\right) / p^{e d} .
$$

This limit exists as a positive real number, as shown by Monsky in [10]. For the maximal ideal $I=\mathfrak{m}$ this number is also called the Hilbert-Kunz multiplicity of the ring $R$ itself.

It is an open question whether the Hilbert-Kunz multiplicity is always rational, and the Hilbert-Kunz function is known only in very special cases. The rationality in the case of a homogeneous coordinate ring $R=k\left[X_{0}, \ldots, X_{N}\right] / \mathfrak{a}$ over a smooth projective curve $C=V_{+}(\mathfrak{a}) \subset \mathbb{P}^{N}$ was shown independently in [4] and [12. This work suggests a more geometric view on the problem of the Hilbert-Kunz function, where stability properties of the syzygy bundle $\operatorname{Syz}\left(X_{0}, \ldots, X_{N}\right)$ on the curve are crucial. For example, if this bundle is strongly semistable, meaning that every Frobenius pull-back of it is again semistable, then the Hilbert-Kunz multiplicity of the cone is given by $e_{H K}(R)=\operatorname{deg}(C)(N+1) / 2 N$ due to [4, Corollary 2.7].

The syzygy bundle $\operatorname{Syz}\left(X_{0}, \ldots, X_{N}\right)$ on the curve is the restriction of the cotangent bundle $\Omega_{\mathbb{P}^{N}}=\mathcal{T}_{\mathbb{P}^{N}}^{\vee}$, as follows from the Euler sequence. What can we say about stability properties of the restriction of the (co)tangent bundle 
to a smooth curve? This is still a difficult question in general, in particular in positive characteristic. In this paper we investigate the case of cones over elliptic curves. The main point which makes elliptic curves more accessible is the fact that a semistable locally free sheaf on an elliptic curve is strongly semistable.

It is not difficult to show that the restriction of the cotangent bundle to an elliptic curve embedded by a complete linear system is stable (Theorem 1.3), hence indecomposable and strongly semistable. We are however also interested in embedding linear systems which are not complete; these linear systems exhibit a rich variety of possible splitting behaviour of the restricted cotangent bundle. We study in the first three sections the vector bundles $E$ on an elliptic curve $C$ which appear as the restriction of the cotangent bundle $\Omega_{\mathbb{P} n}$ for some embedding $\iota: C \rightarrow \mathbb{P}^{n}$. This culminates in the classification result Theorem 3.1, which holds in every characteristic and is of independent interest.

In sections 4-6 we compute the Hilbert-Kunz function of a homogeneous $R_{+^{-}}$ primary ideal $I \subset R=\bigoplus_{n \geq 0} \Gamma\left(C, \mathcal{O}_{C}(n)\right)$, where $C \subset \mathbb{P}^{N}$ is an elliptic curve over an algebraically closed field of positive characteristic. The general result is Theorem 4.4, which describes the Hilbert-Kunz function of $I=\left(f_{1}, \ldots, f_{n}\right)$ as

$$
\varphi(q)=\frac{1}{2 \delta}\left(\sum_{j=1}^{l} \frac{\operatorname{deg}\left(S_{j}\right)^{2}}{\operatorname{rk}\left(S_{j}\right)}-\delta^{2} \sum_{i=1}^{n} d_{i}\right) q^{2}+\gamma(q),
$$

where $\delta=\operatorname{deg}\left(\mathcal{O}_{C}\right), \operatorname{Syz}\left(f_{1}, \ldots, f_{n}\right)(0)=S_{1} \oplus \ldots \oplus S_{l}$ is the decomposition into indecomposable sheaves, $d_{i}=\operatorname{deg}\left(f_{i}\right)$ and $\gamma(q)$ is an eventually periodic function.

In section 5 and 6 we specialize this result to the computation of the HilbertKunz function of an ideal generated by the linear forms $\left(X_{0}, \ldots, X_{N}\right) \subseteq$ $\Gamma\left(C, \mathcal{O}_{C}(1)\right)$, which define the embedding $C \subset \mathbb{P}^{N}$. If this ideal corresponds to a complete linear system - that is $\left(X_{0}, \ldots, X_{N}\right)=\Gamma\left(C, \mathcal{O}_{C}(1)\right)-$, then the semistability result for the restricted cotangent bundle mentioned above yields the Hilbert-Kunz function of the normal homogeneous coordinate rings over an elliptic curve. This gives a new proof of a theorem of FakhruddinTrivedi ([6]), which itself generalizes earlier results of Monsky and BuchweitzChen in the case of a plane cubic curve ([10], [5]).

In the final section 6 we deduce from the classification result for the restricted cotangent bundle the possible Hilbert-Kunz functions for elliptic space curves $C \subset \mathbb{P}^{3}$.

This paper was in part written when the first mentioned author was guest at the Freie Universität Berlin in february 2004. He would like to thank this institution for its hospitality. Furthermore we would like to thank the DFGSchwerpunkt "Globale Methoden in der komplexen Geometrie" for financial support. 


\section{The tangent BUndle of PROJECTIVELy nORMAL CURVES}

Throughout this section $C$, denotes a smooth projective elliptic curve over an algebraically closed field $k$. Recall that a vector bundle is called indecomposable if it is not the direct sum of two proper subbundles.

The question we will discuss in the following sections is the following: Consider an embedding of the elliptic curve $C$ into the projective space $\mathbb{P}^{r}$. This embedding is given by a surjection $\pi: \mathcal{O}_{C}^{\oplus r+1} \rightarrow L$ where $L$ is a very ample line bundle. The Euler sequence of $\mathbb{P}^{r}$ gives that the kernel of $\pi$ is the restriction of $\Omega_{\mathbb{P} r}(1)$ to the curve $C$. We say that a rank $r$ bundle $F$ on $C$ is $a$ restricted twisted cotangent bundle, if and only if there exists an embedding $\iota: C \rightarrow \mathbb{P}^{r}$ such that $\left.F \cong \Omega_{\mathbb{P} r}(1)\right|_{C}$. If $h^{0}(F)>0$, then there exists a linear subspace $\mathbb{P}^{r-h^{0}(F)} \subset \mathbb{P}^{r}$ such that $C$ is contained in this subspace. Therefore, to classify all restricted twisted cotangent bundles it is enough to assume $h^{0}(F)=0$.

In the following Proposition we list the facts about vector bundles on elliptic curves we use thereafter. All these results can be found in Atiyah's article [1. In Tu's paper [13, these results are formulated in the frame work of semistable bundles and their moduli spaces. See also $[8$ for a survey on these results using Fourier-Mukai transforms.

Proposition 1.1. (Properties of vector bundles on an elliptic curve $C$ )

(i) Every vector bundle $E$ on $C$ decomposes into a direct sum of indecomposable ones $E=\oplus E_{i}$.

(ii) There exist indecomposable vector bundles of degree $d$ and rank $r \geq 1$ on $C$. These vector bundles are stable, if and only if $d$ and $r$ are coprime.

(iii) An indecomposable vector bundle is semistable.

(iv) If $E$ is semistable of positive degree $d$, then we have $h^{0}(E)=d$, and $h^{1}(E)=0$. This implies by Serre duality, that for $E$ semistable of negative degree, we have $h^{0}(E)=0$ and $h^{1}(E)=-\operatorname{deg}(E)$.

(v) If $E$ is semistable of degree zero, then we consider the following set: $\Theta_{E}:=\left\{L \in \operatorname{Pic}^{0}(C) \mid h^{0}(E \otimes L) \neq 0\right\}$. The subset $\Theta_{E}$ of $\operatorname{Pic}^{0}(C)$ is non-empty with at most $\operatorname{rk}(E)$ elements. If $E$ is furthermore indecomposable, then $\Theta_{E}$ has exactly one element.

Lemma 1.2. Let $E$ be a semistable vector bundle on the elliptic curve $C$. If $E$ is not isomorphic to the bundle $\mathcal{O}_{C}^{\oplus r}$, then the following holds: $E$ is globally generated, if and only if $\mu(E)>1$.

Proof. In order to study global generatedness, we consider the following long exact sequence for all points $P \in C$ :

$$
H^{0}(E) \stackrel{\psi_{P}}{\longrightarrow} E \otimes k(P) \rightarrow H^{1}(E(-P)) \rightarrow H^{1}(E) \rightarrow 0 .
$$


If $\mu(E)>1$, then $\mu(E(-P))>0$. Thus, by proposition 1.1] (iv), we have $H^{1}(E(-P))=0$ for all points $P$. Thus, all maps $\psi_{P}$ are surjective.

Suppose now, that $E$ is globally generated. In other words, $E$ is a quotient of the semistable bundle $H^{0}(E) \otimes \mathcal{O}_{C}$. Thus, $\mu(E) \geq 0$ with equality only for $E \cong H^{0}(E) \otimes \mathcal{O}_{C}$. Since this case was excluded, we have $H^{1}(E)=0$. Since all the maps $\psi_{P}$ are surjective, we conclude from the above exact sequence that $H^{1}(E(-P))=0$ for all points $P \in C$. Since all line bundles in $\operatorname{Pic}^{0}(C)$ are of type $\mathcal{O}_{C}\left(P_{0}-P\right)$, we conclude from part (v) of proposition [1.1 that $\mu\left(E\left(-P_{0}\right)\right)>0$. Consequently, $\mu(E)>1$.

This lemma yields the stability of the restricted tangent bundle for any embedding of $C$ be a complete linear system:

Theorem 1.3. Let $L$ be a line bundle on $C$ of degree $d \geq 2$. The kernel of the evaluation map $H^{0}(L) \otimes \mathcal{O}_{C} \rightarrow L$ is stable of degree $-d$ and rank $d-1$.

Proof. Denote the kernel of the evaluation map with $K$. By construction, we have $h^{0}(K)=0$. Since the rank and the degree of $K$ are coprime, semistability implies stability. Assume that $K$ is not semistable. Let $K_{1}$ be a subsheaf of maximal slope. This implies that $K_{1}$ is semistable. On the one hand, we have $h^{0}\left(K_{1}\right)=0$, because $K_{1}$ is a subbundle of $K$. On the other hand, we have $\mu\left(K_{1}\right)>\mu(K)=-1-\frac{1}{d-1}$. Since the rank of $K_{1}$ is less then $d-1$, we conclude $\mu\left(K_{1}\right) \geq-1$.

Dualizing the embedding $K_{1} \rightarrow K \rightarrow H^{0}(L) \otimes \mathcal{O}_{C}$, we obtain that $E:=$ $K_{1}^{\vee}$ is globally generated, and by Serre duality $h^{1}(E)=0$. However, since $\mu(E)=-\mu\left(K_{1}\right) \leq 1$, this contradicts the assertion of lemma 1.2.

\section{EMBEDding VECTOR BUNDLES}

Let $E$ be a vector bundle on $C$ which is globally generated and satisfies $h^{1}(E)=0$, then $V:=H^{0}(E)$ is a $k$-vector space of dimension $d=\operatorname{deg}(E)$. The surjection $p: V \otimes \mathcal{O}_{C} \rightarrow E$ defines a morphism $\tilde{p}: C \rightarrow \operatorname{Grass}(V, r)$, where $\operatorname{Grass}(V, r)$ is the Grassmannian variety of $r$ dimensional quotients of $V$. We next investigate when this morphism is an embedding. To do so, we consider the decompostion of $E$ into indecomposable bundles $E=\oplus E_{i}$. We consider now the decompostion of $E=E_{\mu<2} \oplus E_{\mu=2} \oplus E_{\mu>2}$ with the three summands defined by

$$
E_{\mu<2}:=\bigoplus_{\mu\left(E_{i}\right)<2} E_{i}, \quad E_{\mu=2}:=\bigoplus_{\mu\left(E_{i}\right)=2} E_{i}, \quad \text { and } E_{\mu>2}:=\bigoplus_{\mu\left(E_{i}\right)>2} E_{i} .
$$

The next lemma investigates, whether $\tilde{p}$ is an embedding.

Lemma 2.1. Let $E$ be a globally generated vector bundle of degree d and rank $r$ with $h^{1}(E)=0$. We denote by $V$ its space of global sections. The morphism $\tilde{p}: C \rightarrow \operatorname{Grass}(V, r)$ corresponding to the surjection $p: V \otimes \mathcal{O}_{C} \rightarrow E$ is an 
embedding unless $E=E_{\mu=2}$, and $E_{\mu=2}$ is isomorphic to $L^{\oplus \operatorname{rk}(E)}$ for a line bundle $L \in \operatorname{Pic}^{2}(C)$.

Proof. Since $E$ is globally generated, we have $h^{0}(E(-P))=d-r$. Analogously to [7, Proposition IV.3.1], we see that $\tilde{p}$ is an embedding, if and only if for every two points $P, Q \in C$ (including the case $P=Q$ ) the inequality $h^{0}(E(-P-Q))<d-r$ holds.

Suppose that $\tilde{p}$ does not define an embedding. We conclude that there exists two point $P, Q \in C$ such that $h^{0}(E(-P-Q))=h^{0}(E(-P))$. This implies that we have three equalities $h^{0}\left(E_{\mu<2}(-P-Q)\right)=h^{0}\left(E_{\mu<2}(-P)\right)$, $h^{0}\left(E_{\mu=2}(-P-Q)\right)=h^{0}\left(E_{\mu=2}(-P)\right)$, and $h^{0}\left(E_{\mu>2}(-P-Q)\right)=h^{0}\left(E_{\mu>2}(-P)\right)$. However by proposition [1.1 (iv), we conclude that $h^{0}\left(E_{\mu>2}(-P-Q)\right)=$ $h^{0}\left(E_{\mu>2}(-P)\right)-\operatorname{rk}\left(E_{\mu>2}\right)$. This yields $\operatorname{rk}\left(E_{\mu>2}\right)=0$. Hence, $E_{\mu>2}=0$. Analogously we deduce that $E_{\mu<2}=0$.

This yields that $E=E_{\mu=2}$. Consquently we have $\operatorname{deg}(E)=2 r$, and $h^{0}(E(-P)=$ $r$. If $h^{0}(E(-P-Q))=r$, then we have a semistable sheaf of degree zero with $r$ global sections. This implies $E(-P-Q) \cong \mathcal{O}_{C}^{\oplus r}$. Thus, $E \cong\left(\mathcal{O}_{C}(P+Q)\right)^{\oplus r}$, as stated.

On the other hand, we see that for $E \cong\left(\mathcal{O}_{C}(P+Q)\right)^{\oplus r}$ the corresponding morphism $\tilde{p}$ is $2: 1$ onto its image. Reversing the above arguments, we see that $\tilde{p}$ defines an embedding otherwise.

Suppose now that $\tilde{p}: C \rightarrow \operatorname{Grass}(V, r)$ defines an embedding. If this is the case, then we will call $E$ an embedding vector bundle. If the dimension of $V$ equals $r+1$, then we are in the dual situation of theorem [1.3. If $W \subset V$ is a subspace of dimension $r+1$, then we obtain a rational map $\tilde{p}_{W}: C-->\operatorname{Grass}(W, r)$.

We decide next whether $\tilde{p}_{W}$ is a regular morphism to, or even an embedding into $\operatorname{Grass}(W, r)$. We need the following dimension lemma:

Lemma 2.2. Let $V$ be a k-vector space of dimension d. Moreover, fix integers $r, l$, and $k$ satisfying the inequalities

$$
1<r<d-2, \quad 0 \leq k \leq d-r, \quad \text { and } \quad l \geq 1 .
$$

For a subspace $V^{\prime}$ of $V$ of codimension $r+k$, we consider the following subset $Z$ of the Grassmannian $\operatorname{Grass}(r+1, V)$ of $r+1$ dimensional subspaces of $V$ :

$$
Z:=\left\{W \subset V \mid \operatorname{dim}(W)=r+1, \quad \text { and } \quad \operatorname{dim}\left(W \cap V^{\prime}\right) \geq l\right\} .
$$

This $Z$ is a closed subset of $\operatorname{Grass}(r+1, V)$ of codimension $l^{2}+l k-l$.

Proof. This is a standard dimension computation and can be done as follows. Consider the projection $\pi: V \rightarrow V^{\prime \prime}:=V / V^{\prime}$. First, we choose a $(r+1-l)$ dimensional subspace $W^{\prime \prime}$ of $V^{\prime \prime}$. Secondly, we choose $W$ to be an $(r+1)$ dimensional subspace of $\pi^{-1}\left(W^{\prime \prime}\right)$. Using the fact that the dimension of 
the Grassmannian of $m$ dimensional subspaces of an $n$ dimensional space is $m(n-m)$, the lemma follows immediately.

As a consequence, we obtain the following lemma, telling us that most subspaces $W$ of $V$ define a regular morphism $\tilde{p}_{W}$.

Corollary 2.3. If a rank $r$ vector bundle $E$ on a curve $C$ is globally generated with $h^{0}(E)>r+1$, then the $r+1$ dimensional subspaces $W$ of $H^{0}(E)$, which don't generate E, form a proper closed subset of the Grassmannian $\operatorname{Grass}\left(r+1, H^{0}(E)\right)$.

Proof. For a point $P \in C$ we have that $H^{0}(E(-P))$ is of codimension $r$ in $H^{0}(E)$. If $W$ does not generate $E$ at $P$, then we must have $\operatorname{dim}(W \cap$ $\left.H^{0}(E(-))\right) \geq 2$. By lemma 2.2 these $W$ form a closed subscheme of codimension 2. Considering all points $P$ of $C$, we obtain a one dimensional family of codimension two subschemes of $\operatorname{Grass}\left(r+1, H^{0}(E)\right)$.

For a globally generated sheaf $E$ on the elliptic curve $C$, we define the following two numbers:

$$
a_{E}:=\min _{P, Q \in C} h^{0}(E(-P-Q)), \quad \text { and } \quad b_{E}:=\max _{P, Q \in C} h^{0}(E(-P-Q)) .
$$

Lemma 2.4. For a globally generated vector bundle $E$ of rank $r$ on an elliptic curve $C$ we have:

(i) $h^{0}(E)-2 \cdot r \leq a_{E} \leq b_{E} \leq h^{0}(E)-r$;

(ii) The set $U$ of all pairs $(P, Q) \in C \times C$ with $h^{0}(E(-P-Q))=a_{E}$ is Zariski open and nonempty.

(iii) If $a_{E}<b_{E}$, then the complement of $U$ in $C \times C$ consists of a finite union of fibers of the addition map $+: C \times C \rightarrow C$.

Proof. (i) is trivial. (ii) is just the semicontinuity theorem (see [7, Theorem III.12.8]). To prove (iii) we note that for $P+Q=P^{\prime}+Q^{\prime}$, we have $\mathcal{O}_{C}(-P-$ $Q) \cong \mathcal{O}_{C}\left(-P^{\prime}-Q^{\prime}\right)$. Thus, $E(-P-Q) \cong E\left(-P^{\prime}-Q^{\prime}\right)$. However note that $E(-P-Q)$ and $E\left(-P^{\prime}-Q^{\prime}\right)$ are different subsheaves of $E$.

We use these numbers to define two conditions:

We say that a globally generated vector bundle $E$ of rank $r$ satisfies condition $(*)$, if $a_{E}=0$, or $a_{E}<h^{0}(E)-r-2$ holds, and condition $(* *)$, if $b_{E}=0$, or $b_{E}<h^{0}(E)-r-1$ holds.

Using these two conditions and the dimension formula from lemma 2.2 we obtain the following:

Corollary 2.5. Let $E$ be an embedding vector bundle of rank $r \geq 3$ with $h^{1}(E)=0$ on an elliptic curve $C$. If $E$ satisfies the conditions $(*)$ and $(* *)$, then the general $(r+1)$-dimensional subspace $W$ of $H^{0}(E)$ is associated to an embedding $\tilde{p}_{W}: C \rightarrow \mathbb{P}\left(W^{\vee}\right)$. 
Proof. We have seen in corollary 2.3 that a general subspace $W$ of dimension $r+1$ in $H^{0}(E)$ generates $E$. If $W$ does not define an embedding, there must be two points $P$ and $Q$ in $C$ such that $W \cap H^{0}(E(-P))=W \cap H^{0}(E(-Q))$. This implies that $W \cap H^{0}(E(-P-Q))$ is of dimension one. Now we see that lemma 2.2 and the conditions $(*)$, and $(* *)$ yield that those $W$, for which there exists a pair $(P, Q) \in C^{2}$ with $W \cap H^{0}(E(-P-Q))$ is not zero, form a proper closed subscheme of $\operatorname{Grass}\left(r+1, H^{0}(E)\right)$.

Lemma 2.6. Let $E$ be an embedding vector bundle of rank $\operatorname{rk}(E) \geq 3$ with $h^{1}(E)=0$. If $E$ does not satisfy the condition $(*)$, then $E$ is isomorphic to the direct sum $E_{1} \oplus L$, where $E_{1}$ is a stable vector bundle of rank $\operatorname{rk}(E)-1$ and degree $\operatorname{rk}(E)$, and $L$ is a line bundle of degree $\operatorname{deg}(E)-\operatorname{rk}(E) \geq 3$.

Proof. We decompose $E$ into the direct sum $E=E_{\mu<2} \oplus E_{\mu=2} \oplus E_{\mu>2}$. Since by parts (iv) and (v) of proposition 1.1 we have $h^{0}(E(-P-Q))=$ $h^{0}\left(E_{\mu>2}(-P-Q)\right)$ for two general points $P$ and $Q$, we deduce from $a_{E}>0$ that $E_{\mu>2} \neq 0$. Since $E$ is an embedding bundle, we must have $a_{E}<h^{0}(E)-$ $\operatorname{rk}(E)$. To violate condition $(*)$ we have to consider the following two cases:

Case 1: $a_{E}=h^{0}(E)-\operatorname{rk}(E)-1$.

If $E_{\mu<2}$ were trivial, then we would have $a_{E}=h^{0}(E)-2 \operatorname{rk}(E)$ which would imply $E$ is a line bundle. Thus, $E_{\mu<2}$ is nontrivial. From lemma 1.2 we conclude $\operatorname{deg}\left(E_{\mu<2}\right)>\operatorname{rk}\left(E_{\mu<2}\right)$. We have now $1=\left(\operatorname{deg}\left(E_{\mu<2}\right)-\operatorname{rk}\left(E_{\mu<2}\right)\right)+$ $\operatorname{rk}\left(E_{m u=2}\right)+\operatorname{rk}\left(E_{m u>2}\right)$. In this sum of nonnegative integers the first and the last summand are positive which is a contradiction. Thus, the only possibility is the remaining

Case 2: $a_{E}=h^{0}(E)-\operatorname{rk}(E)-2$.

As before, we deduce the equality $2=\left(\operatorname{deg}\left(E_{\mu<2}\right)-\operatorname{rk}\left(E_{\mu<2}\right)\right)+\operatorname{rk}\left(E_{m u=2}\right)+$ $\operatorname{rk}\left(E_{m u>2}\right)$, with the first and last summand positive. Hence, we must have $\operatorname{deg}\left(E_{\mu<2}\right)-\operatorname{rk}\left(E_{\mu<2}\right)=1$, and $E_{\mu>2}$ is a line bundle.

If $E_{\mu<2}=\bigoplus_{i=1}^{l} E_{i}$ is the decomposition into its indecomposable components, the $\operatorname{deg}\left(E_{i}\right)>\operatorname{rk}\left(E_{i}\right)$ has to hold for each component which implies immediately that $E_{\mu<2}$ is indecomposable of $\operatorname{rank} \operatorname{rk}(E)-1$ and degree $\operatorname{rk}(E)$.

Lemma 2.7. Let $E$ be an embedding vector bundle of $\operatorname{rank} r=\operatorname{rk}(E) \geq 3$ with $h^{1}(E)=0$. If $E$ does not satisfy the condition (**), then $E$ is isomorphic to one of the following vector bundles

(i) $E_{1} \oplus L^{\oplus r_{2}}$

$E_{1}$ is stable of rank $\left(\operatorname{rk}(E)-r_{2}\right)>2, r_{2}>0, \operatorname{deg}\left(E_{1}\right)=\operatorname{rk}\left(E_{1}\right)+1$, and $L \in \operatorname{Pic}^{2}(C)$;

(ii) $L^{\oplus(r-1)} \oplus M$

$L$ and $M$ are line bundles with $\operatorname{deg}(L)=2$ and $\operatorname{deg}(M) \geq 3$;

(iii) $L^{\oplus(r-1)} \oplus M$

$L$ and $M$ are line bundles of degree two with $L \nsubseteq M$; 
(iv) $L^{\oplus(r-2)} \oplus\left(L \otimes E_{2}\right)$

$L$ is a line bundle of degree two, and $E$ is the nontrivial extension bundle in $\operatorname{Ext}^{1}\left(\mathcal{O}_{C}, \mathcal{O}_{C}\right)$.

Proof. Since $E$ is an embedding bundle, we have $b_{E}<h^{0}(E)-r$. Thus, to violate the condition $(* *)$, we must have $0<b_{E}=h^{0}(E)-r-1$. We consider the direct sum composition $E=E_{\mu<2} \oplus E_{\mu=2} \oplus E_{\mu>2}$. Analogously to the proof of lemma 2.6. we conclude that

$$
b_{E} \leq h^{0}(E)-r-\left(\operatorname{deg}\left(E_{\mu<2}\right)-\operatorname{rk}\left(E_{\mu<2}\right)\right)-\operatorname{rk}\left(E_{\mu>2}\right) .
$$

Hence, at least one of the bundles $E_{\mu<2}$ or $E_{\mu>2}$ is trivial. Let $P$ and $Q$ be two points of the curve $C$ such that $h^{0}(E(-P-Q))=b_{E}$. We distinguish the three cases:

Case 1: $E_{\mu<2} ¥ 0$.

As in case 2 of lemma 2.6, we conclude that $E_{\mu<2}$ is stable of rank $r_{1}$ and of degree $r_{1}+1$. Thus, $E_{\mu=2}$ turns out to be a bundle of rank $r_{2}=r-r_{1}$, which satisfies $h^{0}\left(E_{\mu=2}(-P-Q)\right)=r_{2}$. Knowing that $E_{\mu=2}$ is semistable of slope zero with $r_{2}$ global sections, we deduce that $E_{\mu=2}(-P-Q) \cong \mathcal{O}_{C}^{\oplus r_{2}}$. Thus, we are in case (i) of the lemma.

Case 2: $E_{\mu>2} \not 0$.

From the above inequality, we deduce that $E_{\mu>2}$ is a line bundle and, as in case 1, we obtain $h^{0}\left(E_{\mu=2}(-P-Q)\right)=\operatorname{rk}\left(E_{\mu=2}\right)$. Thus, the assumption of case 2 yields case (ii) of the lemma.

Case 3: $E=E_{\mu=2}$.

From $h^{0}(E(-P-Q))=r-1$, we deduce that $E(-P-Q)$ contains $\mathcal{O}_{C}^{\oplus r-1}$ as a subbundle. We consider the short exact sequence

$$
0 \rightarrow \mathcal{O}_{C}^{\oplus r-1} \rightarrow E(-P-Q) \rightarrow M \rightarrow 0 .
$$

$M$ is a line bundle of degree zero. If $M$ is not isomorphic to $\mathcal{O}_{C}$, then $\operatorname{Ext}^{1}\left(M, \mathcal{O}_{C}\right)=0$ and this sequence splits. Hence $M \nsubseteq \mathcal{O}_{C}$ implies that we are in case (iii). If $M \cong \mathcal{O}_{C}$, then the above short exact sequence has to be non split because $h^{0}(E)=r-1$. Thus, we are in the remaining case (iv) of our lemma.

Lemma 2.8. Let $E$ be an embedding vector bundle with $h^{1}(E)=0$ of rank $r \geq 3$. If $E$ does not satisfy condition $(*)$, then for all surjections $p: \mathcal{O}_{C} \otimes$ $W \rightarrow E$, where $W$ is a vector space of dimension $r+1$, the corresponding morphism $\tilde{p}: C \rightarrow \operatorname{Grass}(W, r)$ is not an embedding.

Proof. By lemma 2.6. we know that $E$ is isomorphic to $E_{1} \oplus L$ with $L$ a line bundle, and $E_{1}$ stable of degree $r$. Suppose that $p: \mathcal{O}_{C} \otimes W \rightarrow E$ is surjective for $W$ of dimension $r+1$. Consider the composition $\mathcal{O}_{C} \otimes W \rightarrow E \rightarrow E_{1}$. Since $h^{0}\left(E_{1}\right)=r$, we deduce that there exists a nontrivial section $s$ of $L$ in $W$. Since $\operatorname{deg}(L) \geq 3$, we have two points $P$ and $Q$ in $C$ (possibly $P=Q$ ) such that $s \in H^{0}(L(-P-Q))$. However, this means $W \cap H^{0}(E(-P-Q))$ is of dimension one. Thus, $\tilde{p}$ does not separate $P$ and $Q$. 
Lemma 2.9. Let $E$ be an embedding vector bundle with $h^{1}(E)=0$ of rank $r \geq 3$. If $E$ does not satisfy condition (**), then for all surjections $p$ : $\mathcal{O}_{C} \otimes W \rightarrow E$, where $W$ is a vector space of dimension $r+1$, the corresponding morphism $\tilde{p}: C \rightarrow \operatorname{Grass}(W, r)$ is not an embedding.

Proof. We need to examine the four cases of lemma 2.7. We keep the notation of that lemma, and consider an arbitrary surjection $p: \mathcal{O}_{C} \otimes W \rightarrow E$ where $W$ is a vector space of dimension $r+1$. We consider $W$ as a subspace of $H^{0}(E)$. In order to show that $\tilde{p}$ is not an embedding, we have to find two points $P$ and $Q$ in $C$ such that $H^{0}(E(-P-Q)) \cap W$ is one-dimensional. This implies that $\tilde{p}$ does not separate these two points.

Case (i): $E \cong E_{1} \oplus L^{\oplus r_{2}}$.

Consider the resulting morphism $p_{1}: W \rightarrow H^{0}\left(E_{1}\right)$. Its kernel is a subspace of dimension at least $r_{2}$. Take $W^{\prime} \subset W$ to be an $r_{2}$ dimensional subspace of $W$ in the kernel of $p_{1}$. We have $W^{\prime} \subset H^{0}\left(L^{\oplus r_{2}}\right)$. The corresponding morphism $q: \mathcal{O}_{C} \otimes W^{\prime} \rightarrow L^{\oplus r_{2}}$ cannot be surjective. Thus, there exists a point $P \in C$ and a nontrivial section $s \in W^{\prime}$ with $s \in H^{0}\left(L^{\oplus r_{2}}(-P)\right)$. There exists a unique point $Q \in C$ such that $L(-P) \cong \mathcal{O}_{C}(Q)$. This implies that $s$ also lies in $H^{0}\left(L^{\oplus r_{2}}(-P-Q)\right)$. Thus, $s \in W \cap H^{0}(E(-P-Q))$.

Case (ii): $E \cong L^{\oplus r-1} \oplus M$.

Let $L \cong \mathcal{O}_{C}\left(Q_{1}+Q_{2}\right)$. For a point $P \in C$, there exists a unique point $\psi(P)$ such that $L \cong \mathcal{O}_{C}(P+\psi(P))$. Indeed, putting $\psi(P)=Q_{1}+Q_{2}-P$, we obtain this point. For a point $P$, we consider the kernel of the map $W \rightarrow E \otimes k(P)$. Since $E$ is globally generated by $W$, this is spanned by a global section $s$ of $E(-P)$. The global section $s$ yields two global sections $s_{1} \in H^{0}\left(L(-P)^{\oplus r-1}\right)$ and section $s_{2} \in H^{0}(M(-P))$. Since $h^{0}(L(-P))=1$, the section $s_{1}$ vanishes in $\psi(P)$, too. Thus, to show that $\mathcal{O}_{C} \otimes W \rightarrow E$ does not define an embedding, it suffices to show that there exists at least one point $P$ such that the global section $s_{2} \in H^{0}(M(-P))$ also vanishes at $\psi(P)$. To do so, we consider the two projections $p$ and $q$ from $C \times C$ to the components

$$
C \stackrel{q}{\leftarrow} C \times C \stackrel{p}{\rightarrow} C
$$

From the short exact sequence $0 \rightarrow K \rightarrow \mathcal{O}_{C} \otimes W \rightarrow E \rightarrow 0$ on $C$, we construct the following diagram on $C \times C$

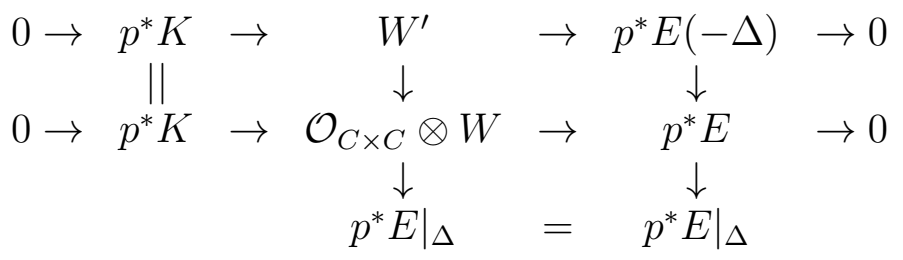

with $\Delta$ denoting the diagonal in the product $C \times C$. Applying the functor $q_{*}$ to the middle column, we obtain that $q_{*} W^{\prime} \cong K$. We obtain a morphism $q^{*} K \rightarrow q_{*}\left(p^{*} M(-\Delta)\right)$. This morphism assigns in any point $P$ of $C$ the global section $s_{2}$ of $M(-P)$. To decide whether this section vanishes at $\psi(P)$, we 
just have to intersect the divisors $\psi(\Delta)=\{(P, \psi(P)) \mid P \in C\}$ with the vanishing divisor $D$ of $q^{*} K \rightarrow p^{*} M(-\Delta)$. Up to numerical equivalence, we obtain $D=\operatorname{deg}(M) F_{p}+(\operatorname{deg}(M)+2 r-2) F_{q}-\Delta$, where $F_{p}$ (resp. $\left.F_{q}\right)$ denote a fiber of $p$ (resp. q). Since D. $\psi(\Delta)=2 \operatorname{deg}(M)+2 r-6>0$, we have points $P$ such that $s_{2}$ vanishes in $\psi(P)$ as well, which contradicts the embedding.

Case (iii) and (iv): There exist two line bundles $L$ and $M$ of degree two, and a short exact sequence $0 \rightarrow L^{\oplus r-1} \rightarrow E \rightarrow M \rightarrow 0$.

Considering the kernel of $W \rightarrow H^{0}(M)$ we find again an $r-1$ dimensional subspace $W^{\prime}$ of global sections of $L^{\oplus r-1}$ in $W$. Now we proceed as in case (i).

\section{Classifying the Restriction of the Cotangent Bundles to ELLIPTIC CURVES}

Now we have everything at hand to give a classification of all restricted twisted cotangent bundles. We remark that such a bundle $F$ is the direct sum $\mathcal{O}_{C}^{\oplus u} \oplus F^{\prime}$ where $F^{\prime}$ is a restricted twisted cotangent bundle without global sections. Therefore, we content ourselves with the classification of all those without global sections.

Theorem 3.1. Let $F$ be a vector bundle on an elliptic curve $C$ of rank $r \geq 3$. Assume furthermore that $h^{0}(F)=0$. Let $F=\bigoplus_{i=1}^{l} F_{i}$ be the decomposition of $F$ into its indecomposable components. Assume that we have $\mu\left(F_{1}\right) \geq$ $\mu\left(F_{2}\right) \geq \ldots \geq \mu\left(F_{l}\right)$. The bundle $F$ is a restricted twisted cotangent bundle, if and only if the conditions (i)-(iv) hold.

(i) The biggest slope $\mu\left(F_{1}\right)$ satisfies $\mu\left(F_{1}\right)<-1$;

(ii) $F$ is not isomorphic to the direct sum $F_{1} \oplus M$ with $M$ a line bundle and $F_{1}$ stable of degree $-r$.

(iii) $F$ is not isomorphic to $F_{1} \oplus L^{\oplus r_{2}}$ with $0<r_{2} \leq r-2, \operatorname{deg}(L)=-2$, and $F_{1}$ stable of degree $r_{2}-r-1$.

(iv) There exists no exact sequence $0 \rightarrow L^{\oplus r-1} \rightarrow F \rightarrow M \rightarrow 0$ where $L$ is a line bundle of degree -2 and $M$ a line bundle of degree $\operatorname{deg}(M) \leq$ -2 .

Proof. If $\iota: C \rightarrow \mathbb{P}^{n}$ is an embedding, then the pull back of the Euler sequence yields $0 \rightarrow \iota^{*} \Omega_{\mathbb{P}^{n}}(1) \rightarrow \mathcal{O}_{C} \otimes V \rightarrow \mathcal{O}_{C}(1) \rightarrow 0$. The morphism $\iota$ is dual to the morphism $\iota^{\vee}: C \rightarrow \operatorname{Grass}\left(V^{\vee}, n\right)$ defined by the surjection $\mathcal{O}_{C} \otimes V^{\vee} \rightarrow\left(\iota^{*} \Omega_{\mathbb{P} n}(1)\right)^{\vee}$. Thus, $\iota^{\vee}$ is an embedding too. Consequently we have that the bundle $E=\left(\iota^{*} \Omega_{\mathbb{P}^{n}}(1)\right)^{\vee}$ is an embedding vector bundle which defines via the surjection of a vector space of dimension $\operatorname{rk}(E)+1$ an embedding into projective space. Now the result follows from the sufficient (Corollary 2.5) and necessary (Lemma 2.8 and Lemma 2.9) conditions (*) and $(* *)$ and the classification of all globally generated vector bundles which do not satisfy these conditions (Lemma 2.6] and Lemma 2.7). 
Let us classify all rank three vector bundles which are restricted twisted cotangent bundles. There are only three cases for the ranks of the decomposition $F=\bigoplus_{i=1}^{l} F_{i}$ into indecomposable bundles.

Case $(i): l=1$, i.e. $F$ is indecomposable.

$F$ is a restricted twisted cotangent bundle, if and only if $\operatorname{deg}(F) \leq-4$.

Case (ii): $l=2$, i.e. $F$ is the direct sum of a line bundle $L$ and a rank two vector bundle $F_{1}$.

$F$ is a restricted cotangent bundle if $\operatorname{deg}\left(F_{1}\right) \leq-4$, and $\operatorname{deg}(L) \leq-2$. In the case that in both inequalities the equality holds, we must furthermore demand that $\operatorname{Hom}\left(F_{1}, L\right)=0$.

Case (iii): $l=3$, i.e. $F$ is a direct sum of three line bundles $L_{1} \oplus L_{2} \oplus L_{3}$. $F$ is a restricted cotangent bundle if $\operatorname{deg}\left(L_{i}\right) \leq-2$, and any two of these line bundles which are of degree -2 , are not isomorphic to each other.

\section{The Hilbert-Kunz FunCtion OVER AN ELLIPTIC CURVE}

In this section we compute the Hilbert-Kunz function of an $R_{+}$-primary homogeneous ideal $I \subseteq R$, where $R=\bigoplus_{n \in \mathbb{N}} \Gamma(C, \mathcal{O}(n))$ is the graded section ring associated to a very ample invertible sheaf $\mathcal{O}(1)$ on an elliptic curve $C$. Homogeneous ideal generators $I=\left(f_{1}, \ldots, f_{n}\right)$ of degree $d_{i}=\operatorname{deg}\left(f_{i}\right)$ give rise to the short exact sequence on $C$,

$$
0 \longrightarrow \operatorname{Syz}\left(f_{1}, \ldots, f_{n}\right)(m) \longrightarrow \bigoplus_{i=1}^{n} \mathcal{O}\left(m-d_{i}\right) \stackrel{f_{i}}{\longrightarrow} \mathcal{O}(m) \longrightarrow 0
$$

which relates the computation of the Hilbert-Kunz function to the computation of the global sections of $\operatorname{Syz}\left(f_{1}, \ldots, f_{n}\right)(m)$ and of its Frobenius pull-backs. We first need the following Lemma.

Lemma 4.1. Let $C$ denote an elliptic curve over an algebraically closed field $k$ of positive characteristic $p$ together with a fixed very ample invertible sheaf $\mathcal{O}(1)$ of degree $\delta=\operatorname{deg}(C)$. Let $S$ denote an indecomposable sheaf on $C$. Set $\nu=-\mu(S) / \delta=-\operatorname{deg}(S) / \operatorname{rk}(S) \delta$. Let $\sigma$ and $\rho$ denote natural numbers such that $\sigma \leq \nu<\rho$. Write $\lceil q \nu\rceil=q \nu+\pi(q)$ with an eventually periodic function $\pi(q)$. Then

$$
\begin{aligned}
\sum_{m=q \sigma}^{q \rho-1} h^{0}\left(S^{q}(m)\right)= & \frac{q^{2}}{2 \delta}\left(\frac{\operatorname{deg}(S)^{2}}{\operatorname{rk}(S)}+2 \rho \operatorname{deg}(S) \delta+\rho^{2} \operatorname{rk}(S) \delta^{2}\right) \\
& -q \frac{\delta}{2}\left(\rho \operatorname{rk}(S)+\frac{\operatorname{deg}(S)}{\delta}\right)+\pi(q)(1-\pi(q)) \operatorname{rk}(S) \frac{\delta}{2} \\
& +h^{1}\left(S^{q}(\lceil q \nu\rceil)\right)
\end{aligned}
$$

Proof. An indecomposabe sheaf on an elliptic curve is strongly semistable. For $m<\lceil q \nu\rceil$ we have $m<-q \mu(S) / \delta$ and therefore

$$
\operatorname{deg}\left(S^{q}(m)\right)=q \operatorname{deg}(S)+m \operatorname{rk}(S) \delta<0 .
$$


Since $S^{q}$ is semistable we have $h^{0}\left(S^{q}(m)\right)=0$ for $m<\lceil q \nu\rceil$. So the sum starts with $m=\lceil q \nu\rceil$.

For $m>\lceil q \nu\rceil$ we find that $h^{1}\left(S^{q}(m)\right)=0$ by semistability and Serre duality. Hence by Riemann-Roch we get

$$
\begin{aligned}
\sum_{m=\lceil q \nu\rceil}^{q \rho-1} h^{0}\left(S^{q}(m)\right)= & \sum_{m=\lceil q \nu\rceil}^{q \rho-1} \operatorname{deg}\left(S^{q}(m)\right)+h^{1}\left(S^{q}(\lceil q \nu\rceil)\right) \\
= & \sum_{m=\lceil q \nu\rceil}^{q \rho-1}(q \operatorname{deg}(S)+m \operatorname{rk}(S) \delta)+h^{1}\left(S^{q}(\lceil q \nu\rceil)\right) \\
= & q(q \rho-\lceil q \nu\rceil) \operatorname{deg}(S) \\
& +(q \rho(q \rho-1)-\lceil q \nu\rceil(\lceil q \nu\rceil-1)) \frac{\operatorname{rk}(S) \delta}{2} \\
& +h^{1}\left(S^{q}(\lceil q \nu\rceil)\right) .
\end{aligned}
$$

We write $\lceil q \nu\rceil=q \nu+\pi(q)$. The first summand is $\left(q^{2} \rho-q^{2} \nu-q \pi(q)\right) \operatorname{deg}(S)$ and the second summand is

$$
\left(q^{2}\left(\rho^{2}-\nu^{2}\right)-q(\rho-\nu)-2 q \nu \pi(q)-\pi(q)(\pi(q)-1)\right) \frac{\operatorname{rk}(S) \delta}{2} .
$$

We regroup and use $\nu=-\operatorname{deg}(S) / \operatorname{rk}(S) \delta$ to get the stated result.

Remark 4.2. Let $S$ denote an indecomposable bundle as in lemma 4.1 . set $\nu=-\operatorname{deg}(S) / \operatorname{rk}(S) \delta$. Then we have defined $\pi(q)$ by the condition $\lceil q \nu\rceil=q \nu+\pi(q)$. Since $\lceil a / b\rceil=a / b+(-a \bmod \mathrm{b}) / \mathrm{b}$, we have

$$
\pi(q)=(q \operatorname{deg}(S) \bmod \operatorname{rk}(\mathrm{S}) \delta) / \operatorname{rk}(\mathrm{S}) \delta .
$$

This is eventually a periodic function. In important cases, e.g. if $S$ is an indecomposable syzygy bundle, the degree $\operatorname{deg}(S)$ is a multiple of the curve degree, say $\operatorname{deg}(S)=u \delta$; then we can write $\pi(q)=(q u \bmod \operatorname{rk}(\mathrm{S})) / \operatorname{rk}(\mathrm{S})$ and the length of the periodicity is bounded by $\operatorname{rk}(S)$.

Remark 4.3. Suppose again the situation of the previous lemma. If $q \nu=$ $-q \operatorname{deg}(S) / \operatorname{rk}(S) \delta$ is not an integer, then $\operatorname{deg}\left(S^{q}(\lceil q \nu\rceil)\right)>0$ and the $h^{1}$-term vanishes. So suppose that $q \nu$ is an integer so that $S^{q}(q \nu)$ has degree 0. Due to $S^{q p}(q p \nu)=\left(S^{q}(q \nu)\right)^{p}$ we see that $\operatorname{deg}\left(S^{q}(q \nu)\right)=0$ for all $q \gg 0$. Since $S^{q}(q \nu)$ is strongly semistable, we have a decomposition $S^{q}(q \nu)=G_{1} \oplus \ldots \oplus G_{t}$ with indecomposable bundles of degree 0 . Every $G_{j}$ equals $G_{j}=F \otimes L$, where $L$ is an invertible sheaf of degree 0 and where $F$ is the unique indecomposable sheaf of degree 0 and $\operatorname{rank} \operatorname{rk}(F)=\operatorname{rk}\left(G_{j}\right)$ with a non-trivial global section (see proposition $1.1 \mathrm{v}(\mathrm{v})$ ).

If the Hasse-invariant of the elliptic curve is 0 , then $F^{q}$ is trivial for $q \gg 0$. In this case $S^{q}(q \nu)$ is a direct sum of line bundles of degree $0, q \gg 0$. For a line bundle $L$ either $L^{q}=\mathcal{O}$ for some $q$ or not. Therefore for $q \gg 0$ we have $S^{q}(q \nu)=\mathcal{O}^{u} \oplus L_{1} \oplus \ldots \oplus L_{m}$, where $L_{j}$ are line bundles such that no $q$-power of them gets trivial. The number of sections of $S^{q}(q \nu)$ as well as the dimension of $H^{1}\left(S^{q}(\lceil q \nu\rceil)\right)$ is then eventually constant $=u$. 
If the Hasse-invariant of the elliptic curve is 1 , then there exists also a decomposition $S^{q}(q \nu)=\mathcal{O}^{u} \oplus G_{1} \oplus \ldots \oplus G_{t}$ such that the $G_{j}$ are strongly stable. If $G_{j}=F \otimes L$, then $\left(G_{j}\right)^{p}=F \otimes L^{p}$, and the dimension of the global sections of $S^{q}(q \nu)$ is again eventually constant.

Theorem 4.4. Let $k$ denote an algebraically closed field of positive characteristic $p$. Let $C \subset \mathbb{P}^{N}$ denote an elliptic curve of degree $\delta=\operatorname{deg}(C)=$ $\operatorname{deg}(\mathcal{O}(1) \mid C)$. Set $R=\bigoplus_{n \in \mathbb{N}} \Gamma(C, \mathcal{O}(n))$. Let I denote a homogeneous $R_{+}$primary ideal in $R$ given by homogeneous ideal generators $I=\left(f_{1}, \ldots, f_{n}\right)$ of degree $d_{i}=\operatorname{deg}\left(f_{i}\right)$. Let $\operatorname{Syz}\left(f_{1}, \ldots, f_{n}\right)(0)=S_{1} \oplus \ldots \oplus S_{l}$ denote the decomposition of the syzygy bundle into indecomposable locally free sheaves on $C$. Set $\nu_{j}=-\operatorname{deg}\left(S_{j}\right) / \operatorname{rk}\left(S_{j}\right) \delta$ Write $\left\lceil q \nu_{j}\right\rceil=q \nu_{j}+\pi_{j}(q)$ with the eventually periodic functions $\pi_{j}(q)$. Then the Hilbert-Kunz function of $I$ is

$$
\varphi(q)=e_{H K}(I) q^{2}+\gamma(q)
$$

where

$$
e_{H K}(I)=\frac{1}{2 \delta}\left(\sum_{j=1}^{l} \frac{\operatorname{deg}\left(S_{j}\right)^{2}}{\operatorname{rk}\left(S_{j}\right)}-\delta^{2} \sum_{i=1}^{n} d_{i}^{2}\right)
$$

is a rational number and where

$$
\gamma(q)=\sum_{j=1}^{l} \frac{\operatorname{rk}\left(S_{j}\right) \delta}{2} \pi_{j}(q)\left(1-\pi_{j}(q)\right)+\sum_{j=1}^{l} h^{1}\left(S_{j}^{q}\left(\left\lceil q \nu_{j}\right\rceil\right)\right)-n+1 .
$$

If $q \nu_{j}$ is not a natural number, then the $h^{1}$-term is 0 . In any case the $h^{1}$-term is eventually constant and $\gamma(q)$ is an eventually periodic function.

Proof. We look at the sequence

$$
0 \longrightarrow \operatorname{Syz}\left(f_{1}, \ldots, f_{n}\right)(m) \longrightarrow \bigoplus_{i=1}^{n} \mathcal{O}\left(m-d_{i}\right) \stackrel{f_{1}, \ldots, f_{n}}{\longrightarrow} \mathcal{O}(m) \longrightarrow 0
$$

where $\operatorname{Syz}\left(f_{1}, \ldots, f_{n}\right)(m) \cong S_{1}(m) \oplus \ldots \oplus S_{l}(m)$. The pull-back of this short exact sequence under the $e$-th absolute Frobenius morphism $F^{e}: C \rightarrow C$ yields

$$
0 \longrightarrow\left(F^{e}\left(\operatorname{Syz}\left(f_{1}, \ldots, f_{n}\right)\right)\right)(m) \longrightarrow \bigoplus_{i=1}^{n} \mathcal{O}\left(m-q d_{i}\right) \stackrel{f_{1}^{q}, \ldots, f_{n}^{q}}{\longrightarrow} \mathcal{O}(m) \longrightarrow 0
$$

where $\left.F^{e}\left(\operatorname{Syz}\left(f_{1}, \ldots, f_{n}\right)\right)\right)(m) \cong S_{1}^{q}(m) \oplus \ldots \oplus S_{l}^{q}(m)$. We have $\varphi(q)=$ $\sum_{m=0}^{\infty} \lambda\left(\left(R / I^{[q]}\right)_{m}\right)$ and

$$
\lambda\left(\left(R / I^{[q]}\right)_{m}\right)=h^{0}(\mathcal{O}(m))-\sum_{i=1}^{n} h^{0}\left(\mathcal{O}\left(m-q d_{i}\right)\right)+h^{0}\left(\operatorname{Syz}\left(f_{1}^{q}, \ldots, f_{n}^{q}\right)(m)\right) .
$$

We sum these expressions from $m=0$ up to $m=q \rho, \rho \gg 0$. We apply Lemma 4.1 to these sheaves. Lets denote for fixed numbers $\sigma \ll 0$ and $\rho \gg 0$ the expressions from Lemma 4.1 for an indecomposable sheaf $T$ by $\Phi(T)$. Then we have to compute $\Phi(\mathcal{O})-\sum_{i=1}^{n} \Phi\left(\mathcal{O}\left(-d_{i}\right)\right)+\sum_{j=1}^{l} \Phi\left(S_{j}\right)$. We have written down the sum of the $\operatorname{deg}(T)^{2}$-terms. Since the rank and the degree are additive in a short exact sequence, the corresponding terms 
sum up to 0 . We have written down the periodicity terms and the $h^{1}$-term coming from $T=\operatorname{Syz}\left(f_{1}, \ldots, f_{n}\right)$. For the invertible sheaves $\mathcal{O}(d)$ there is no periodicity term, since $\nu=-\operatorname{deg} \mathcal{O}(-d) / \delta=d$ is always rational, and the $h^{1}$-term is always 1 . This together gives the result.

Remark 4.5. It is true in general for an $R_{+}$-primary homogeneous ideal $I$ in a normal two-dimensional standard-graded domain $R$ that the Hilbert-Kunz function has the form $\varphi(q)=e_{H K}(I) q^{2}+\gamma(q)$ with a rational Hilbert-Kunz multiplicity $e_{H K}(I)$ and a bounded function $\gamma(q)$, see [3]. Moreover, if the base field is the algebraic closure of a finite field, then $\gamma(q)$ is an eventually periodic function. This finiteness condition is not necessary in the elliptic case; for higher genus this question is open, see also [9].

Corollary 4.6. Let $k$ denote an algebraically closed field of positive characteristic p. Let $C \subset \mathbb{P}^{N}$ denote an elliptic curve of degree $\delta=\operatorname{deg}(C)=$ $\operatorname{deg}(\mathcal{O}(1) \mid C)$. Set $R=\bigoplus_{n \in \mathbb{N}} \Gamma(C, \mathcal{O}(n))$. Let $I$ denote a homogeneous $R_{+}$primary ideal in $R$ given by homogeneous ideal generators $I=\left(f_{1}, \ldots, f_{n}\right)$ of degree $d_{i}=\operatorname{deg}\left(f_{i}\right)$ and suppose that the syzygy bundle $\operatorname{Syz}\left(f_{1}, \ldots, f_{n}\right)$ is semistable. Then the Hilbert-Kunz function of $I$ is

$$
\varphi(q)=e_{H K}(I) q^{2}+\gamma(q)
$$

where

$$
e_{H K}(I)=\frac{\delta}{2}\left(\frac{\left(\sum_{i=1}^{n} d_{i}\right)^{2}}{n-1}-\sum_{i=1}^{n} d_{i}^{2}\right)
$$

and

$$
\begin{aligned}
\gamma(q)= & \frac{\delta}{2} \frac{-q \sum_{i=1}^{n} d_{i} \bmod (\mathrm{n}-1)}{n-1}\left(1-\frac{-q \sum_{i=1}^{n} d_{i} \bmod (\mathrm{n}-1)}{n-1}\right) \\
& +h^{1}\left(\operatorname{Syz}\left(f_{1}^{q}, \ldots, f_{n}^{q}\right)\left(\left\lceil\frac{q \sum_{i=1}^{n} d_{i}}{n-1}\right\rceil\right)\right)-n+1 .
\end{aligned}
$$

Proof. This follows easily from theorem 4.4 using the relationships

$$
\operatorname{deg}\left(\operatorname{Syz}\left(f_{1}, \ldots, f_{n}\right)(0)\right)=-\delta \sum_{i=1}^{n} d_{i}, \nu=\frac{\delta \sum_{i=1}^{n} d_{i}}{(n-1) \delta}=\frac{\sum_{i=1}^{n} d_{i}}{n-1}
$$

and

$$
\pi(q)=\frac{\left(-q \delta \sum_{i=1}^{n} d_{i} \bmod (\mathrm{n}-1) \delta\right)}{(n-1) \delta}=\frac{-q \sum_{i=1}^{n} d_{i} \bmod \mathrm{n}-1}{n-1}
$$

\section{The Hilbert-Kunz FunCtion of AN ideAl Generated By LineAR FORMS}

We bring now the results of the previous sections together to compute the Hilbert-Kunz function of an ideal $I \subset R$ generated by an embedding linear system, where $R=\bigoplus_{n \in \mathbb{N}} \Gamma(C, \mathcal{O}(n))$ is the graded section ring associated to a very ample invertible sheaf $\mathcal{O}(1)$ on an elliptic curve $C$ over an algebraically 
closed field $k$ of positive characteristic $p$. If $C \subset \mathbb{P}^{N}=\operatorname{Proj} K\left[X_{0}, \ldots, X_{N}\right]$ is the corresponding embedding, then $I=\left(X_{0}, \ldots, X_{N}\right)$ and the syzygy bundle $\operatorname{Syz}\left(X_{0}, \ldots, X_{N}\right)=\Omega_{\mathbb{P}^{N} \mid C}$. Theorem 4.4 gives at once the following general corollary. If we want to say more about the behavior of the HilbertKunz function then we need more information about the restriction of the cotangent bundle.

Corollary 5.1. Let $k$ denote an algebraically closed field of positive characteristic $p$. Let $C \subset \mathbb{P}^{N}=\operatorname{Proj} k\left[X_{0}, \ldots, X_{N}\right]$ denote an elliptic curve of degree $\delta=\operatorname{deg}(C)=\operatorname{deg}(\mathcal{O}(1) \mid C)$. Set $R=\bigoplus_{n \in \mathbb{N}} \Gamma(C, \mathcal{O}(n))$. Let $\operatorname{Syz}\left(X_{0}, \ldots, X_{N}\right)=\Omega_{\mathbb{P}^{N}} \mid C=S_{1} \oplus \ldots \oplus S_{l}$ denote the decomposition of the restricted cotangent bundle into indecomposable locally free sheaves on $C$. Set $\nu_{j}=-\operatorname{deg}\left(S_{j}\right) / \operatorname{rk}\left(S_{j}\right) \delta$ Write $\left\lceil q \nu_{j}\right\rceil=q \nu_{j}+\pi_{j}(q)$ with the eventually periodic functions $\pi_{j}(q)$. Then the Hilbert-Kunz function of the ideal $I=\left(X_{0}, \ldots, X_{N}\right) \subset R$ is

$$
\varphi(q)=e_{H K}(I) q^{2}+\gamma(q)
$$

where the Hilbert-Kunz multiplicity is

$$
e_{H K}(I)=\frac{1}{2 \delta}\left(\sum_{j=1}^{l} \frac{\operatorname{deg}\left(S_{j}\right)^{2}}{\operatorname{rk}\left(S_{j}\right)}-\delta^{2}(N+1)\right)
$$

and where

$$
\gamma(q)=\sum_{j=1}^{l} \frac{\operatorname{rk}\left(S_{j}\right) \delta}{2} \pi_{j}(q)\left(1-\pi_{j}(q)\right)+\sum_{j=1}^{l} h^{1}\left(S_{j}^{q}\left(\left\lceil q \nu_{j}\right\rceil\right)\right)-N .
$$

is an eventually periodic function.

Proof. This follows directly from Theorem 4.4 using $n=N+1$.

If the elliptic curve $C \subset \mathbb{P}^{N}$ is embedded by a complete linear system - that is $\left(X_{0}, \ldots, X_{N}\right)=\Gamma(C, \mathcal{O}(1))$ - then we know by theorem 1.3 that the restriction of the cotangent bundle is semistable. Therefore we obtain the following result of Fakhruddin and Trivedi (see [6. Corollary 3.18]) as a corollary.

Theorem 5.2. Let $C \subset \mathbb{P}^{N}$ denote an elliptic curve over an algebraically closed field $k$ of positive characteristic $p$ embedded by a complete linear system $\left|\mathcal{O}_{C}(1)\right|$. Then the Hilbert-Kunz function of the maximal ideal in the homogeneous coordinate ring $R=k\left[X_{0}, \ldots, X_{N}\right] / \mathfrak{a}$ is given by

$$
\varphi(q)=e_{H K}(R) q^{2}+\gamma(q)
$$

where $e_{H K}(R)=\frac{(N+1)^{2}}{2 N}$ and where

$$
\gamma(q)=\frac{N+1}{2}(-q \bmod \mathrm{N})\left(1-\frac{(-\mathrm{q} \bmod \mathrm{N})}{\mathrm{N}}\right)+\mathrm{h}^{1}\left(\Omega\left(\left\lceil\frac{\mathrm{q}(\mathrm{N}+1)}{\mathrm{N}}\right\rceil\right)\right)-\mathrm{N} .
$$

If $N \neq p^{e}$, then the $h^{1}$-term vanishes. 
Proof. The curve $C \subset \mathbb{P}^{N}$ is projectively normal. Therefore the domain $R=\bigoplus_{n \in \mathbb{N}} \Gamma(C, \mathcal{O}(n))$ is normal and standard-graded, that is generated by finitely many forms of degree one, hence $R \cong k\left[X_{0}, \ldots, X_{N}\right] / \mathfrak{a}$. Due to the assumption, the dimension of the global sections of $\mathcal{O}_{C}(1)=\left.\mathcal{O}_{\mathbb{P}^{N}}(1)\right|_{C}$ is $N+1$. The global dimension and the degree of an ample invertible sheaf on an elliptic curve are the same. The degree of $\mathcal{O}_{C}(1)$ is the degree of the embedding $C \subset \mathbb{P}^{N}$, hence $\operatorname{deg}(C)=N+1$ The restriction of the tangent bundle $\mathcal{T}_{\mathbb{P}^{N}}$ to $C$ is semistable due to theorem 1.3 and therefore strongly semistable, hence we apply corollary 5.1 with $l=1$. The rank of the syzygy bundle is $N$ and its degree is $-(N+1)^{2}$. This gives for the Hilbert-Kunz multiplicity $e_{H K}(\mathfrak{m})=$

$=\frac{1}{2 \delta}\left(\frac{\operatorname{deg}(\operatorname{Syz})^{2}}{\operatorname{rk}(\operatorname{Syz})}-(N+1)^{2}\right)=\frac{1}{2(N+1)}\left(\frac{(N+1)^{4}}{N}-(N+1)^{3}\right)=\frac{(N+1)^{2}}{2 N}$.

The $O\left(q^{0}\right)$-term is $\gamma(q)=\frac{N(N+1)}{2} \pi(q)(1-\pi(q))+h^{1}(\Omega(\lceil q \nu\rceil))-N$ where $\nu=-\operatorname{deg}(\Omega \mid C) / \operatorname{rk}(\Omega) \delta=(N+1) / N$. From $\lceil q \nu\rceil=\lceil q(N+1) / N\rceil=$ $q(N+1) / N+\pi(q)$ it follows that $\pi(q)=(-q(N+1) \bmod \mathrm{N})=(-\mathrm{q} \bmod \mathrm{N})$. This gives the result.

In the following corollaries we write down the Hilbert-Kunz functions in the complete case for $N=2,3,4$. For $N=2$, the case of a plane smooth cubic, we get the following Theorem of Buchweitz-Chen (see [5, Theorem 4]).

Corollary 5.3. Let $C \subset \mathbb{P}^{2}$ denote a plane elliptic curve over an algebraically closed field of characteristic $p \geq 3$. Let $R=k[X, Y, Z] /(F)$ denote its homogeneous coordinate ring. Then the Hilbert-Kunz function of $R$ is

$$
\varphi(q)=\frac{9}{4} q^{2}-\frac{5}{4}
$$

Proof. Since $q$ is odd we have to insert $(-q \bmod 2)=1$ in the formula of Theorem 5.2. This yields

$$
\varphi(q)=\frac{9}{4} q^{2}+\frac{3}{2}\left(1-\frac{1}{2}\right)-2=\frac{9}{4} q^{2}-\frac{5}{4} .
$$

The next corollary treats the case of an elliptic space curve embedded by a complete linear system. The homogeneous coordinate ring is a complete intersection ring $R=K\left[X_{0}, X_{1}, X_{2}, X_{3}\right] /(f, g)$, where $f$ and $g$ are two quadrics.

Corollary 5.4. Let $C \subset \mathbb{P}^{3}$ denote an elliptic curve over an algebraically closed field of characteristic $p \neq 3$ embedded by a complete linear system in projective space. Then the Hilbert-Kunz function of the homogeneous coordinate ring $R=k\left[X_{0}, X_{1}, X_{2}, X_{3}\right] /(f, g)$ is

$$
\varphi(q)=\frac{8}{3} q^{2}-\frac{5}{3}
$$


Proof. The formula in Theorem 5.2 yields

$$
\varphi(q)=\frac{8}{3} q^{2}+2\left((-q \bmod 3)\left(1-\frac{(-\mathrm{q} \bmod 3)}{3}\right)\right)-3 .
$$

It does not matter whether $q \bmod 3=1$ or 2 , the big bracket yields always $2 / 3$.

Corollary 5.5. Let $C \subset \mathbb{P}^{4}$ denote an elliptic curve over an algebraically closed field of characteristic $p \neq 2$ embedded by a complete linear system. Then the Hilbert-Kunz function of the homogeneous coordinate ring $R$ is

$$
\varphi(q)=\frac{25}{8} q^{2}-\frac{17}{8} .
$$

Proof. The formula in Theorem 5.2 yields

$$
\varphi(q)=\frac{25}{8} q^{2}+\frac{5}{2}\left((-q \bmod 4)\left(1-\frac{(-\mathrm{q} \bmod 4)}{4}\right)\right)-4 .
$$

The big bracket yields always $3 / 4$.

In the previous corollaries we have excluded the case $N=p^{e}$ to avoid the cohomological term $h^{1}\left(\Omega\left(\left\lceil\frac{q(N+1)}{N}\right\rceil\right)\right)$ from theorem 5.2. We now deal with the case $N=p=2$ and we recover the main result of [1] due to Monsky. Recall the notion of the Hasse invariant (or $p$-rank) of an elliptic curve in positive characteristic. The Hasse-invariant is 0 or 1 according whether the Frobenius morphism $H^{1}\left(C, \mathcal{O}_{C}\right) \rightarrow H^{1}\left(C, \mathcal{O}_{C}\right)$ it the zero map or bijective.

Corollary 5.6. Let $C \subset \mathbb{P}^{2}$ denote a plane elliptic curve over an algebraically closed field of characteristic 2 . Let $R=K[X, Y, Z] /(F)$ denote the homogeneous coordinate ring. Then the Hilbert-Kunz function of $R$ is

$$
\varphi(1)=1, \varphi(2)=8, \varphi\left(2^{e}\right)=\frac{9}{4} 2^{2 e} \text { for } e \geq 2
$$

for Hasse-invariant 0 and

$$
\varphi(1)=1, \varphi(2)=7, \varphi\left(2^{e}\right)=\frac{9}{4} 2^{2 e}-1 \text { for } e \geq 2
$$

for Hasse-invariant 1.

Proof. By theorem 5.2 we have $\varphi(1)=1$ and

$$
\varphi(q)=\frac{9}{4} q^{2}+h^{1}\left(\operatorname{Syz}\left(X^{q}, Y^{q}, Z^{q}\right)\left(\frac{3}{2} q\right)\right)-2
$$

for $q=2^{e} \geq 2$. Therefore we have to show for Hasse-invariant 0 that $h^{1}\left(\operatorname{Syz}\left(X^{q}, Y^{q}, Z^{q}\right)\left(\frac{3}{2} q\right)\right)=1$ for $q=2$ and $=2$ for $q \geq 4$, and for Hasseinvariant 1 that $h^{1}\left(\operatorname{Syz}\left(X^{q}, Y^{q}, Z^{q}\right)\left(\frac{3}{2} q\right)\right)=0$ for $q=2$ and $=1$ for $q \geq 4$. The bundle $\operatorname{Syz}\left(X^{q}, Y^{q}, Z^{q}\right)\left(\frac{3}{2} q\right)$ has rank two and its determinant is $\mathcal{O}_{C}$, therefore it is isomorphic to its dual, and we can replace $h^{1}$ by $h^{0}$.

Up to an automorphism we may assume that $C$ is given by an equation $X^{3}+Y^{3}+Z^{3}+\lambda X Y Z=0$. Due to [7, Proposition IV.4.21] the curve has 
Hasse invariant 0 if and only if $\lambda=0$. Moreover, for $\lambda^{3}=1$ it is easy to see that the point $(1, \lambda, \lambda)$ is a singular point on the curve, hence $\lambda^{3} \neq 1$.

First assume that $\lambda=0$, so we are dealing with the Fermat cubic $X^{3}+$ $Y^{3}+Z^{3}=0$. This curve equation yields at once the syzygy $(X, Y, Z)$ for $\left(X^{2}, Y^{2}, Z^{2}\right)$ of degree 3 and hence the short exact sequence

$$
0 \longrightarrow \mathcal{O}_{C} \longrightarrow \operatorname{Syz}\left(X^{2}, Y^{2}, Z^{2}\right)(3) \longrightarrow \mathcal{O}_{C} \longrightarrow 0 \text {. }
$$

This shows that the first Frobenius pull-back of the restricted tangent bundle is not stable (though semistable). It is clear that $h^{0}\left(C, \operatorname{Syz}\left(X^{2}, Y^{2}, Z^{2}\right)(3)\right)=$ 1. Therefore this sequence does not split and it is given by a non-trivial cohomology class $c \in H^{1}\left(C, \mathcal{O}_{C}\right)$. Since the Hasse-invariant is 0 , the Frobenius pull-back of the short exact sequence splits and hence

$$
\operatorname{Syz}\left(X^{q}, Y^{q}, Z^{q}\right)(m) \cong \mathcal{O}(m-3 q / 2) \oplus \mathcal{O}(m-3 q / 2)
$$

for $q \geq 4$. Therefore the correction term is $h^{0}\left(C, \operatorname{Syz}\left(X^{q}, Y^{q}, Z^{q}\right)(3 q / 2)\right)=2$.

We consider now the case of Hasse-invariant is 1 , which means that $\lambda \neq 0$, $\lambda^{3} \neq 1$. It is clear that $H^{0}\left(C, \operatorname{Syz}\left(X^{2}, Y^{2}, Z^{2}\right)(3)\right)=0$. So we look at the syzygies $H^{0}\left(C, \operatorname{Syz}\left(X^{4}, Y^{4}, Z^{4}\right)(6)\right)$. Multiplying the curve equation with $X^{3}+Y^{3}+Z^{3}$ yields

$$
\begin{aligned}
& \left(X^{3}+Y^{3}+Z^{3}\right)\left(X^{3}+Y^{3}+Z^{3}+\lambda X Y Z\right) \\
= & X^{6}+Y^{6}+Z^{6}+\lambda X^{4} Y Z+\lambda X Y^{4} Z+\lambda X Y Z^{4} \\
= & X^{4}\left(X^{2}+\lambda Y Z\right)+Y^{4}\left(Y^{2}+\lambda X Z\right)+Z^{4}\left(Z^{2}+\lambda X Y\right)
\end{aligned}
$$

hence $\left(X^{2}+\lambda Y Z, Y^{2}+\lambda X Z, Z^{2}+\lambda X Y\right)$ is a global syzygy for $\left(X^{4}, Y^{4}, Z^{4}\right)$ of degree 6 . The corresponding short exact sequence is

$$
0 \longrightarrow \mathcal{O}_{C} \longrightarrow \operatorname{Syz}\left(X^{4}, Y^{4}, Z^{4}\right)(6) \longrightarrow \mathcal{O}_{C} \longrightarrow 0 \text {. }
$$

We claim that this sequence does not split. To see this we show that the space of global syzygyies of degree 6 for $X^{4}, Y^{4}, Z^{4}$ is one-dimensional. Such a syzygy is given by a multiple of the curve equation fulfilling

$$
H\left(X^{3}+Y^{3}+Z^{3}+\lambda X Y Z\right) \in\left(X^{4}, Y^{4}, Z^{4}\right),
$$

where $\operatorname{deg} H=3$. The polynomial $H$ is a linear combination of the 10 monomials of degree 3 . The monomial $X Y Z$ cannot occur, since the product $\lambda X^{2} Y^{2} Z^{2}$ cannot arise in another way. The products with $X^{3}, Y^{3}, Z^{3}$ yield $X^{3} Y^{3}$ etc., and these monomials do not occur in another way. If $H=$ $a X^{3}+b Y^{3}+c Z^{3}$ we arrive at the syzygy from above.

Hence we may assume that $H$ is a linear combination of monomials of type $(1,2)$, say $H=a X^{2} Y+b X Y^{2}+c X Z^{2}+d X^{2} Z+e Y Z^{2}+f Y^{2} Z$. Multiplying with the curve equation yields (modulo $\left(X^{4}, Y^{4}, Z^{4}\right)$ ) the terms

$$
\begin{gathered}
(b+e \lambda) X Y^{2} Z^{3}+(c+f \lambda) X Y^{3} Z^{2}+(d+b \lambda) X^{2} Y^{3} Z \\
+(a+c \lambda) X^{2} Y Z^{3}+(e+d \lambda) X^{3} Y Z^{2}+(f+a \lambda) X^{3} Y^{2} Z
\end{gathered}
$$


The conditions $b+e \lambda=0, d+b \lambda=0$ and $e+d \lambda=0$ yield $e=e \lambda^{3}$ (and $\left.f=f \lambda^{3}\right)$. Since $\lambda^{3} \neq 1$ we see $e=f=0$ and hence all coefficients are 0 . This proves the claim.

Since the Hasse-invariant is $\neq 0$ it follows that also all the Frobenius pullbacks of the short exact sequences do not split. From this it follows that $h^{0}\left(C, \operatorname{Syz}\left(X^{q}, Y^{q}, Z^{q}\right)(3 q / 2)\right)=1$ for $q \geq 4$.

\section{Elliptic SPACE CURVES}

In this last section we deal with the Hilbert-Kunz function of an elliptic space curve $C \subset \mathbb{P}^{3}$. The degree of $C$ may be every number $\delta \geq 4$. For $\delta=4$ the curve is embedded by a complete linear system and the associated graded section ring $R=\bigoplus_{n \in \mathbb{N}} \Gamma\left(C, \mathcal{O}_{C}(1)\right)$ is normal and standard-graded. This is not true for $\delta \geq 5$ and the following theorem computes the Hilbert-Kunz function of the ideal $\left(X_{0}, X_{1}, X_{2}, X_{3}\right)$ of the graded section ring $R$, not of the homogeneous coordinate ring. We call this the Hilbert-Kunz function of the curve $C \subset \mathbb{P}^{3}$ embedded by the linear system $\left(X_{0}, X_{1}, X_{2}, X_{3}\right)=$ $\left.\Gamma\left(\mathbb{P}^{3}, \mathcal{O}(1)\right)\right|_{C} \subseteq \Gamma(C, \mathcal{O}(1))$. The classification from section 3 yields the following list of Hilbert-Kunz functions.

Theorem 6.1. Let $C \subset \mathbb{P}^{3}$ denote an elliptic space curve of degree $\delta$. Then there exist the following three possibilities for the Hilbert-Kunz function of $C$, depending on the splitting behavior of $\left.\Omega_{\mathbb{P}^{3}}\right|_{C}$.

(i) Suppose that $\Omega \mid C$ is indecomposable. Then

$$
\varphi(q)=\frac{2 \delta}{3} q^{2}+\frac{\delta}{3}-3 .
$$

for $p \neq 3$ and

$$
\varphi(q)=\frac{2 \delta}{3} q^{2}+h^{1}\left(\operatorname{Syz}\left(X_{0}^{q}, X_{1}^{q}, X_{2}^{q}, X_{3}^{q}\right)\left(\frac{4 q}{3}\right)\right)-3
$$

for $p=3($ for $q \neq 1)$.

(ii) Suppose that $\Omega \mid C \cong S \oplus M$, where $S$ is indecomposable of rank two and $M$ is invertible. Set $\nu_{1}=-\operatorname{deg}(S) / 2 \delta$ and $\nu_{2}=-\operatorname{deg}(M) / \delta$ and write $\left\lceil q \nu_{j}\right\rceil=q \nu_{j}+\pi_{j}(q), j=1,2$. Then

$$
\begin{aligned}
\varphi(q)= & \frac{1}{2 \delta}\left(\frac{\operatorname{deg}(S)^{2}}{2}+\operatorname{deg}(M)^{2}-4 \delta^{2}\right) q^{2}+\delta \pi_{1}(q)\left(1-\pi_{1}(q)\right)+\frac{\delta}{2} \pi_{2}(q)\left(1-\pi_{2}(q)\right) \\
& +h^{1}\left(S^{q}\left(\left\lceil\frac{-q \operatorname{deg}(S)}{2 \delta}\right\rceil\right)\right)+h^{1}\left(M^{q}\left(\left\lceil\frac{-q \operatorname{deg}(M)}{\delta}\right\rceil\right)\right)-3
\end{aligned}
$$

(iii) Suppose that $\Omega \mid C \cong M_{1} \oplus M_{2} \oplus M_{3}$ with three invertible sheaves $M_{1}$, $M_{2}$ and $M_{3}$. Set $\nu_{j}=-\operatorname{deg}\left(M_{j}\right) / \delta$ and write $\left\lceil q \nu_{j}\right\rceil=q \nu_{j}+\pi_{j}(q)$, $j=1,2,3$. Then

$$
\varphi(q)=\frac{\operatorname{deg}\left(M_{1}\right)^{2}+\operatorname{deg}\left(M_{2}\right)^{2}+\operatorname{deg}\left(M_{3}\right)^{2}-4 \delta^{2}}{2 \delta} q^{2}+\frac{\delta}{2}\left(\sum_{j=1}^{3} \pi_{j}(q)\left(1-\pi_{j}(q)\right)\right)
$$




$$
\begin{aligned}
& +h^{1}\left(M_{1}^{q}\left(\left\lceil\frac{-q \operatorname{deg}\left(M_{1}\right)}{\delta}\right\rceil\right)\right)+h^{1}\left(M_{2}^{q}\left(\left\lceil\frac{-q \operatorname{deg}\left(M_{2}\right)}{\delta}\right\rceil\right)\right) \\
& +h^{1}\left(M_{3}^{q}\left(\left\lceil\frac{-q \operatorname{deg}\left(M_{3}\right)}{\delta}\right\rceil\right)\right)-3
\end{aligned}
$$

Proof. The rank of $\Omega \mid C$ is 3 and its degree is $-4 \delta$. We compute the HilbertKunz multiplicity as $e_{H K}=\frac{1}{2 \delta}\left(\sum_{j} \frac{\operatorname{deg}\left(S_{j}\right)^{2}}{\operatorname{rk}\left(S_{j}\right)}-4 \delta^{2}\right)$ (theorem 4.4). This gives in the indecomposable case $\frac{1}{2 \delta}\left(\frac{16 \delta^{2}}{3}-4 \delta^{2}\right)=\delta\left(\frac{8}{3}-2\right)=2 \delta / 3$. In the other cases we just have inserted.

Now we look at the lower term $\gamma(q)$ coming from theorem 4.4. In the indecomposable case we have by remark 4.2

$$
\pi(q)=(q \operatorname{deg}(S) \bmod 3 \delta) / 3 \delta=(-4 \mathrm{q} \bmod 3) / 3=(-\mathrm{q} \bmod 3) / 3 .
$$

For $p \neq 3$ we have $\frac{(-q \bmod 3)}{3}\left(1-\frac{(-q \bmod 3)}{3}\right)=\frac{2}{9}$, for $p=3$ this term is 0 for $q \neq 1$. In cases (ii) and (iii) we have just written down the formula for $\gamma(q)$ coming from theorem 4.4 .

Remark 6.2. Suppose that $C \subset \mathbb{P}^{3}$ is an elliptic space curve of degree $\delta$. If $\Omega \mid C$ is semistable, then the Hilbert-Kunz multiplicity is $2 \delta / 3$. This is automatically true if $\Omega \mid C$ is indecomposable, but this holds also in case (ii) if $\operatorname{deg}(M)=\operatorname{deg}(S) / 2$ and in case (iii) if the degrees of the invertible sheaves $M_{1}, M_{2}$ and $M_{3}$ are equal. If $\Omega \mid C$ is not semistable, then $e_{H K}(C)>2 \delta / 3$. This follows from a direct computation or from [2, Lemma 1.3].

\section{REFERENCES}

[1] M. F. Atiyah. Vector bundles over an elliptic curve. Proc. London Math. Soc., 7:414$452,1957$.

[2] H. Brenner. A characteristic zero Hilbert-Kunz criterion for solid closure in dimension two. To appear in Math. Research Letters, 2004.

[3] H. Brenner. The Hilbert-Kunz function in graded dimension two. ArXiv, 2004.

[4] H. Brenner. The rationality of the Hilbert-Kunz multiplicity in graded dimension two. ArXiv, 2004.

[5] R.-O. Buchweitz and Q. Chen. Hilbert-Kunz functions of cubic curves and surfaces. J. Algebra, 197(1):246-267, 1997.

[6] N. Fakhruddin and V. Trivedi. Hilbert-Kunz functions and multiplicities for full flag varieties and elliptic curves. J. Pure Appl. Algebra, 181(1):23-52, 2003.

[7] R. Hartshorne. Algebraic Geometry. Springer, 1977.

[8] G. Hein and D. Ploog. Stable bundles and Fourier-Mukai transforms on elliptic curves. Preprint Serie A - 11 - 2003, Freie Universität Berlin, 2003.

[9] C. Huneke, M. McDermott, and P. Monsky. Hilbert-Kunz functions for normal rings. Preprint, 2003.

[10] P. Monsky. The Hilbert-Kunz function. Math. Ann., 263:43-49, 1983.

[11] P. Monsky. The Hilbert-Kunz function of a characteristic 2 cubic. J. Algebra, 197(1):268-277, 1997.

[12] V. Trivedi. Semistability and Hilbert-Kunz multiplicity for curves. ArXiv, 2004.

[13] L. W. Tu. Semistable bundles over an elliptic curve. Adv. Math., 98:1-26, 1993. 
Mathematische Fakultät, Ruhr-Universität Bochum, Universitätsstr 150, 44780 Bochum, Germany; Fachbereich Mathematik und Informatik, Freie Universität Berlin, Arnimallee 3, 14195 Berlin, Germany.

E-mail address: Holger.Brenner@ruhr-uni-bochum.de, ghein@math.fu-berlin.de 